\title{
Sistemas SUPLEMENTARES E ALTERNATIVOS DE COMUNiCAÇÃo NAS HABILIDADES EXPRESSIVAS DE UM ALUNO COM PARALISIA CEREBRAL
} AUGMENTATIVE AND ALTERNATIVE SYSTEMS IN THE EXPRESSIVE ABILITIES OF A CEREBRAL PALSY STUDENT

\author{
Débora DELIBERATO ${ }^{1}$
}

RESUMO: o objetivo desta pesquisa foi descrever o uso do sistema de comunicação suplementar e alternativo de um aluno com paralisia cerebral submetido à intervenção. Participou desta investigação um aluno com paralisia cerebral de 10 anos de idade, gênero masculino, que frequentava classe especial para deficientes físicos em uma escola estadual de uma cidade do interior de São Paulo. As atividades programadas foram realizadas duas vezes por semana, durante dois anos, em um Laboratório de Educação Especial de uma Universidade pública. Todas as fitas gravadas descrevendo as atividades desenvolvidas nos atendimentos efetivados durante o processo de avaliação e implementação do recurso de comunicação suplementar e alternativo foram assistidas e descritas, em um protocolo especifico. Com base nas informações dos protocolos, foram selecionadas as sessões com intervalo maior que 20 dias e que continham atividades envolvendo o tabuleiro de comunicação, com o tempo igual ou superior a 20 minutos, durante o primeiro ano da intervenção. As sessões escolhidas foram transcritas na íntegra e, após análise do texto obtido, foram estabelecidas as seguintes categorias: o sistema gráfico auxiliou o aluno, na emissão de estrutura vertical $(56 \%)$, associado à modalidade oral $(14 \%)$ e à modalidade não oral verbal e não verbal (30\%), enquanto o uso do sistema gráfico, em conjunto com outras modalidades, colaborou na ampliação dos enunciados e na possibilidade de o aluno ser compreendido na sua intenção. O emprego de sistemas de comunicação suplementar e alternativo proporcionou ao aluno a ampliação de situações dialógicas efetivas, durante as atividades realizadas na intervenção fonoaudiológica.

PALAVRAS-CHAVE: Educação Especial. Sistemas de comunicação. Habilidades comunicativas. Inclusão. Intervenção.

ABSTRACT: The objective of this research was to describe the use of an augmentative and alternative system for a student with cerebral palsy during intervention. A 10-year-old male student with cerebral palsy participated in this investigation. He attended special classes for people with disability in a public school in a city of the interior of Sao Paulo. The scheduled activities were carried out twice a week for two years at the Laboratory of Special Education of a public University of São Paulo. All of the tapes describing the activities that were filmed during the assessment and implementation of augmentative and alternative communication resource were watched and described using a specific protocol. Based on the information from the protocols, the sessions with intervals greater than 20 days and the ones with activities involving the communication board with the time equal or greater than 20 minutes during the first year of intervention were selected. The chosen sessions were transcribed in full and, after analyzing the text, the following categories were established, according to the stated goal: the graphic system helped the student with utterances of vertical structure (56\%) associated with the oral (14\%) and non-oral and non-verbal form $(30 \%)$, while the use of the graphic system along with other forms collaborated to enhance statements, enabling better understanding of the child's intention. The use of augmentative and alternative communication systems provided the expansion of effective dialogical situations for the student during the activities carried out in speech therapy.

KEYWORDS: Special education. Communication systems. Communicative abilities. Inclusion. Intervention.

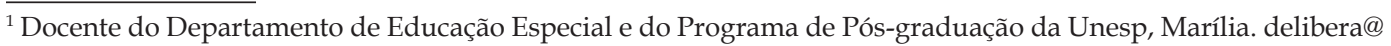
marilia.unesp.br
} 


\section{INTRODUÇão}

A complexidade da estrutura da aquisição e desenvolvimento da linguagem de crianças com deficiência, e sem a possibilidade de falar, tem sido objeto de investigação de diferentes áreas do conhecimento, principalmente dos pesquisadores preocupados com a aquisição das habilidades comunicativas e o uso dos sistemas pictográficos no processo de aprendizagem da leitura e escrita de crianças e jovens usuários de sistemas suplementares e alternativos de comunicação (SMITH, 2003; VON TETZCHNER; GROVE, 2003; DELIBERATO, 2009).

O saber a respeito das questões biológicas e ambientais envolvidas no processamento da aquisição e desenvolvimento da linguagem têm permitido aos pesquisadores entender a dificuldade em estabelecer e padronizar programas de intervenção para as crianças e jovens com deficiência e severa complexidade de comunicação (DELIBERATO, 2009). Quando a família, o professor ou mesmo um profissional da saúde se deparam com alunos com deficiência e severos distúrbios de linguagem, surgem as angústias e a sensação de incapacidade de contatar essas crianças e jovens com o mundo e com as questões pedagógicas (DELIBERATO, 2007; SAMESHIMA, 2011).

A garantia de programas mais adequados à diversidade de cada aluno com pouca ou nenhuma fala funcional poderia viabilizar acesso ao conhecimento, ampliação dos processos interacionais, habilidades comunicativas, educacionais e sociais e, assim, propiciar uma maior independência e favorecer a qualidade de vida das crianças e jovens com deficiência usuários de sistemas suplementares e alternativos de comunicação (BLAU, 1996; DELIBERATO, 2007).

Segundo Von Tetzchner eGrove (2003), os estudos da área de Comunicação Suplementar e Alternativa (CSA) ainda não aprofundaram os detalhes do desenvolvimento da criança usuária dos sistemas suplementares e alternativos de comunicação da perspectiva da linguagem. Os autores alertaram que são escassos os trabalhos que descreveram as questões semânticas, sintáticas e pragmáticas das crianças que utilizaram os sistemas de comunicação suplementar e alternativa, comparando-as com as crianças que adquirem e desenvolvem a linguagem falada ou as crianças que apresentam distúrbios da linguagem falada, mas que possuem a fala como instrumento de comunicação. Nesse contexto, seria possível identificar a falta de estudos a respeito das etapas da aquisição e desenvolvimento da linguagem das crianças e jovens usuários de sistemas suplementares e alternativos de comunicação independente do sistema de representação e do idioma utilizado na comunidade linguística dos mesmos.

A falta de estudos a respeito de como ocorre à aquisição e desenvolvimento da linguagem das crianças que usam os sistemas de CSA acaba gerando uma falta de critérios para a elaboração de programas de intervenção de forma mais direcionada (VON TETZCHNER; GROVE, 2003). 
McCleary e Smith (2006) discutiram que crianças com impedimento da linguagem falada podem explorar diferentes modos de comunicação até conquistarem e encontrarem um sistema ideal e funcional. Entre as possibilidades de comunicação, as autoras identificaram pranchas de comunicação de baixa tecnologia com diferentes símbolos, aparelhos com saída de voz, gestos e sinais manuais. Embora identificassem as modalidades expressivas empregadas pelas crianças, os autores também pontuaram a dificuldade em sistematizar o processo de escolha dos sistemas e recursos a serem usados pelas crianças. Nesse processo de seleção do sistema suplementar e alternativo de comunicação, os autores perceberam que as preferências das próprias crianças e as participações de seus familiares contribuíram para sistematizar a modalidade ou modalidades de comunicação mais funcional (DELIBERATO; MANZINI, 2008).

Higginbotham et al. (2007) encontraram evidências, por meio de pesquisas e do próprio desenvolvimento da área de CSA, de que o acesso às tecnologias de CSA está associado a uma complexa inter-relação entre os aspectos das tecnologias apresentadas pela área, com: habilidades físicas (motor, sensorial, perceptual), habilidades cognitivas e as habilidades linguísticas dos usuários dos sistemas de CSA. Os mesmos autores ainda enfatizaram que as habilidades de comunicação dos diferentes interlocutores com os usuários dos sistemas e a sua capacitação para lidar com as tecnologias de CSA demonstradas contribuíram para o sucesso no estabelecimento do uso dos diferentes materiais (NUNES, 2003b; DELIBERATO; MANZINI, 2008).

Outro aspecto contemplado nas pesquisas a respeito das habilidades comunicativas nos usuários de sistemas pictográficos seria o uso de símbolos únicos para designar uma mensagem mais complexa, ou seja, as crianças e jovens não utilizam, com frequência, a combinação de elementos gráficos para a constituição sintática de sua expressão. Smith e Grove (2003) discutiram que as crianças usuárias de CSA, frequentemente, recorrem a símbolos únicos (fotos, desenhos, figuras) a fim de se comunicarem quando utilizam sistemas suplementares e alternativos de comunicação (estrutura vertical). Os fatores que poderiam contribuir para as crianças utilizarem símbolos únicos seriam: 1) falta de eficiência comunicativa, isto é, com a finalidade de serem mais rápidos, usam mensagens mais curtas (SMITH; GROVE, 2003); 2) um único símbolo poderia representar múltiplos conceitos simultaneamente para uma criança, desta forma não necessitaria usar outros símbolos, como, por exemplo, figuras de uma pessoa comendo um sorvete pode significar: comer, sorvete ou eu quero comer sorvete (SMITH, 1996); 3) os parceiros de comunicação normalmente fazem perguntas que requerem respostas simples de uma única palavra, geralmente "sim" ou "não" (LIGHT; BINGER; SMITH, 1994).

Sameshima e Deliberato (2009) discutiram que tanto a falta de oportunidades para comunicar quanto os tipos de oportunidades que são oferecidos podem colaborar para os baixos índices de produção de mensagens de multissímbolos (estrutura horizontal). As autoras reforçaram que as crianças que 
usam os sistemas de CSA precisam ser ensinadas a como produzir mensagens de multissímbolos nos diferentes contextos naturais.

Pesquisadores e atuantes na área de CSA têm demonstrado a preocupação com a elaboração e sistematização de programas de intervenção nos ambientes de reabilitação e nos ambientes naturais por meio de sistemas suplementares e alternativos de comunicação para garantir habilidades comunicativas funcionais e favorecer a inserção das crianças e jovens com deficiência nos diferentes ambientes (COOK; HUSSEY, 2002).

Neste contexto de discussão, o objetivo deste estudo foi caracterizar o uso de sistemas gráficos de um aluno com paralisia cerebral durante a intervenção baseada em sistemas suplementares e alternativos de comunicação.

\section{Material e MÉtodo}

Este estudo fez parte de uma pesquisa maior a respeito da Análise das habilidades comunicativas de alunos com paralisia cerebral durante o uso de recursos de comunicação suplementares e alternativos, aprovada pelo Comitê de ética pelos pareceres $n^{\circ} 1882 / 2003$ e $n^{\circ} 1883 / 2003$. O critério de seleção dos dados para o presente trabalho foi analisar o perfil do uso dos sistemas gráficos no contexto das demais habilidades expressivas durante um conjunto de etapas de trabalho com os sistemas de comunicação alternativa. O critério de seleção do aluno para esta discussão ocorreu em função do acompanhamento desse aluno por diferentes profissionais da saúdeeeducaçãonas distintasetapasnecessárias para otrabalhocom a comunicação alternativa: avaliação, seleção, implementação e acompanhamento dos sistemas e recursos de comunicação alternativa. No período de realização das atividades selecionadas para esta pesquisa, o aluno selecionado possuía 10 anos de idade; apresentava o diagnóstico neurológico de paralisia cerebral quadriplégica do tipo espástica, em decorrência de anóxia perinatal; locomovia-se por meio de cadeira de rodas; não revelava alteração na acuidade auditiva e/ou visual e não apresentava alteração na deglutição. O aluno frequentava classe especial de uma escola da rede estadual de uma cidade do interior de São Paulo.

A avaliação inicial das habilidades comunicativas da criança foi realizada pela fonoaudióloga e pesquisadora dessa pesquisa por meio de procedimentos direcionados as temáticas da rotina de atividades familiares e escolares. Nessa avaliação inicial foi possível identificar compreensão de ordens simples e complexas e severo distúrbio de expressão oral. As modalidades expressivas empregadas foram: o riso, o sorriso, o olhar, os movimentos de face (diferentes expressões faciais) e de cabeça, o apontar com a mão direita e algumas vocalizações com entonações, principalmente com as vogais /ã/ e / u/. Cabe ressaltar que, segundo a avaliação da professora, o aluno não apresentava deficiência intelectual; porém, era bastante disperso, direcionando a atenção para detalhes do contexto e, consequentemente, tendia a perder o foco principal da interação, momento este em que era necessária maior mediação. 
Quanto às questões escolares, ainda segundo informações do professor, o aluno era lento para desenvolver as atividades preparadas, estava reconhecendo as vogais e somente se comunicava por meio de alguns sons, respondendo com a cabeça para afirmação e negação. Ainda conforme o professor, o aluno tinha um bom potencial, embora relatasse a dificuldade de avaliá-lo, pelo fato de ele não falar.

Como instrumentos de coleta foram empregados sistemas de comunicação suplementar e alternativos de baixa tecnologia (SORO-CAMATS, 2003): objetos, fotos, figuras e figuras do sistema pictográfico PCS - Picture Communication Symbols - e recursos de alta tecnologia, como o programa ImagoAnaVox (CAPOVILLA; DUDUCHI; MACEDO, 1998) e o Boardmaker (JOHNSON, 1994).

O trabalho de avaliação, implementação e acompanhamento da criança foi realizado no Laboratório de Educação Especial de uma Universidade Pública do Estado de São Paulo, com a duração de dois anos. Os atendimentos aconteciam duas vezes na semana e com duração de 60 minutos. Participaram das atividades a terapeuta fonoaudióloga, pesquisadora da presente pesquisa, a família do participante (mãe, pai e irmão) e demais interlocutores: fisioterapeuta, terapeuta ocupacional, psicólogo e alunos do Curso de Pedagogia - Habilitação em Educação Especial. Convém esclarecer que o aluno selecionado realizava atendimento nas áreas de fonoaudiologia, fisioterapia, terapia ocupacional e pedagogia, mas, nas atividades previstas do programa relacionado às ações com a comunicação alternativa, os profissionais eram convidados a participar da atividade em conjunto no mesmo atendimento.

Durante os dois primeiros meses, foi realizada a avaliação dos aspectos sensoriais, perceptivos, motores e linguísticos seguindo as considerações de Deliberato e Manzini (1997). O vocabulário básico da rotina familiar foi inserido no tabuleiro de comunicação suplementar e alternativa durante o processo de avaliação inicial e seleção do sistema suplementar e alternativo de comunicação. No momento em que os demais profissionais iniciaram a participação do programa de implementação e acompanhamento do sistema e dos recursos de comunicação suplementares e alternativos, os novos símbolos foram selecionados e adaptados em conjunto com os profissionais participantes do processo de reabilitação e com o usuário propriamente dito.

Desde o início da implementação do sistema de comunicação suplementar de baixa e alta tecnologia, os atendimentos foram registrados por meio filmagens e do caderno de registro, o que possibilitou acompanhar as mudanças das possibilidades comunicativas adotadas por esse aluno no processo de intervenção (FAGUNDES, 1983). As observações descritas do caderno de registro foram realizadas por alunos do curso de Pedagogia e, após cada descrição o conteúdo, era discutido com a fonoaudióloga, pesquisadora da pesquisa.

Para o presente trabalho, foram selecionadas filmagens que abrangiam o tabuleiro de comunicação suplementar e alternativa, após o seu uso pelo participante, nas atividades do processo de intervenção fonoaudiológica como 
instrumento facilitador da sua comunicação. Dessa forma, a partir do momento em que o tabuleiro de comunicação passou a ser empregado pelo aluno como instrumento de expressão, ficou estabelecido o início de marcador temporal para a seleção do material para análise desta pesquisa. A partir do marcador temporal do uso do sistema de comunicação suplementar e alternativo pelo aluno, foram selecionadas 12 atividades envolvendo o tabuleiro de comunicação suplementar e alternativa (MILLIKIN, 1997). As atividades foram escolhidas com base em 12 sessões fonoaudiológicas, com espaço entre elas de pelo menos 20 dias, correspondendo ações realizadas durante um ano de intervenção por meio do uso de sistemas e recursos de comunicação suplementar e alternativa.

As fitas contendo as atividades selecionadas foram transcritas na íntegra e foram analisados os trechos das transcrições em que o participante utilizava o tabuleiro de comunicação durante 20 minutos consecutivos. Os 20 minutos selecionados e transcritos em cada sessão foram conferidos por duas outras pessoas da área, que não participaram do processo de intervenção com o aluno. As informações do caderno de registro foram incorporadas à transcrição do material da filmagem, as quais, provenientes desse instrumento de coleta, inseriram-se no texto em letra Times versão negrito. As informações do caderno de registro foram significativas para o entendimento do contexto temporal das atividades realizadas no programa e, também, para ampliar as informações obtidas por meio do uso da filmadora.

Após a constituição do texto escrito definitivo, ou seja, após a inserção das informações do caderno de registro no texto obtido por meio das transcrições das filmagens, foram estabelecidas as categorias de análises por meio dos critérios estabelecidos por Bardin (2004) para caracterizar o uso dos sistemas gráficos utilizados perante as diferentes habilidades expressivas utilizadas. Cabe destacar que foram selecionadas as categorias e subcategorias direcionadas ao uso dos sistemas gráficos entre as demais habilidades expressivas utilizadas nas atividades desenvolvidas pelo participante com os interlocutores.

Sendo assim, durante a análise do texto escrito obtido por meio da transcrição das filmagens selecionadas em conjunto com as informações do caderno de registro foram estabelecidas as seguintes categorias e subcategorias:

Habilidades expressivas: qualquer modalidade expressiva da linguagem, utilizada pela criança participante, com intencionalidade e entendida pelo interlocutor, no momento da interação, sendo esta modalidade relacionada a um sistema linguístico mais amplo ou não.

A - Expressão oral: todas as expressões sonoras, utilizadas pela criança, com intenção comunicativa entendida pelo interlocutor, ou seja, as emissões de vogais ou qualquer outro fonema ou ainda sons produzidos pelo corpo. Nesta categoria, foram inseridas as subcategorias: Riso/risada, Vocalizações, Palavras, Emissão ininteligível (não entendida). 
B - Expressão não-oralizada: modalidade de expressões utilizadas pela criança com intenção comunicativa, entendidas pelo interlocutor sem som associado, isto é, sem a emissão de vogais ou qualquer outro fonema, ou ainda sons produzidos pelo corpo. Nesta categoria, foram inseridas as subcategorias: Gesto com as mãos, Movimento com a cabeça, Expressão facial. Convém ressaltar que na subcategoria de gestos com as mãos e movimento com a cabeça foram inseridos o uso do sistema gráfico.

C - Expressões nas modalidades não-orais combinadas: foram consideradas as emissões nas quais a criança utilizou dois ou mais recursos comunicativos nãoorais, diferentes ou da mesma subcategoria, em sequência ou ao mesmo tempo, que resultassem em significados para o interlocutor no momento da interação, como, por exemplo: Expressão facial + movimento com cabeça; Movimento com as mãos + expressão facial + movimento de cabeça entre outras possibilidades.

$D$ - Expressão oral e expressão não-oral: habilidades expressivas nas modalidades orais e não-orais usadas, concomitantemente ou em sequência pela criança, com intenção comunicativa entendida pelo interlocutor. Foram consideradas nesta categoria as habilidades expressivas orais e não-orais ou na sequência não-orais e orais.

Após as categorias e subcategorias serem estabelecidas com os respectivos exemplos, o material foi enviado para dois juízes da área da Educação Especial, a fim de ser avaliado. A realização do índice de concordância teve como finalidade demonstrar se as categorias e subcategorias identificadas pela pesquisadora estavam representadas pelos exemplos dos registros realizados: filmagens e caderno de registro. O parâmetro comparativo aceitável intra e entre juízes, relativo às ocorrências, deveria ser igual ou superior a 70\% (CARVALHO, 1996). A concordância referiu-se à comparação dos dados totais, obtidos pela pesquisadora $(\mathrm{P})$, com os do juiz $\mathrm{A}(\mathrm{P}-\mathrm{A})=90 \%$; pesquisadora com os do juiz $\mathrm{B}(\mathrm{P}-\mathrm{B})=90 \%$ e entre os dados dos juízes entre si $(\mathrm{A}-\mathrm{B})=92,4 \%$.

\section{Resultados E Discussões}

Neste momento serão apresentados os resultados identificados a respeito do uso do sistema gráfico utilizado nos procedimentos selecionados no contexto do processo interacional com os diferentes interlocutores quanto ao número de ocorrências e a organização do uso. No que se refere aos aspectos qualitativos, os exemplos das categorias e subcategorias alvos serão identificados seguindose os critérios da transcrição e submetidos ao destaque negrito, no contexto da situação dialógica. Além do destaque em negrito para a categoria ou subcategoria alvo, também está descrito em itálico a categoria ou subcategoria expressa pelo aluno, nas situações dialógicas determinadas. A categoria ou subcategoria alvo é o conteúdo selecionado para análise no exemplo citado, uma vez que, no mesmo exemplo, seria possível a identificação de outras categorias ou subcategorias. 
Para facilitar a leitura dos exemplos, segue a legenda estabelecida para a transcrição dos exemplos: T1: Terapeuta1; T2: Terapeuta2; A: Aluno; E1: Estagiária 1; E2: Estagiária 2; M: Mãe; P: Pai; I: Irmão; ( ) descrição das ações e gestos utilizados pelos interlocutores; (( )) descrição das intenções gestuais e motoras no contexto do aluno participante: interpretação do uso dos gestos no contexto; [ ] emissões orais do aluno participante; \{\} descrição das intenções das emissões orais no contexto; CAIXA ALTA: indicação da figura selecionada; Entonações orais com significado emitidas pelo aluno participante, compreendidas pelos interlocutores.

Como já foi descrito anteriormente, as atividades selecionadas estavam vinculadas ao emprego do tabuleiro de comunicação suplementar e alternativa, que estava sendo adequado e adaptado durante o processo de intervenção. Ainda que toda a atividade selecionada com o tabuleiro tenha sido transcrita, foram analisados os primeiros 20 minutos de cada atividade.

Durante os 12 procedimentos realizados com o tabuleiro de comunicação suplementar e alternativa foi possível identificar que o aluno utilizou o recurso com menor frequência nos procedimentos 9, 11 e 12, como pode ser observado no Gráfico 1:

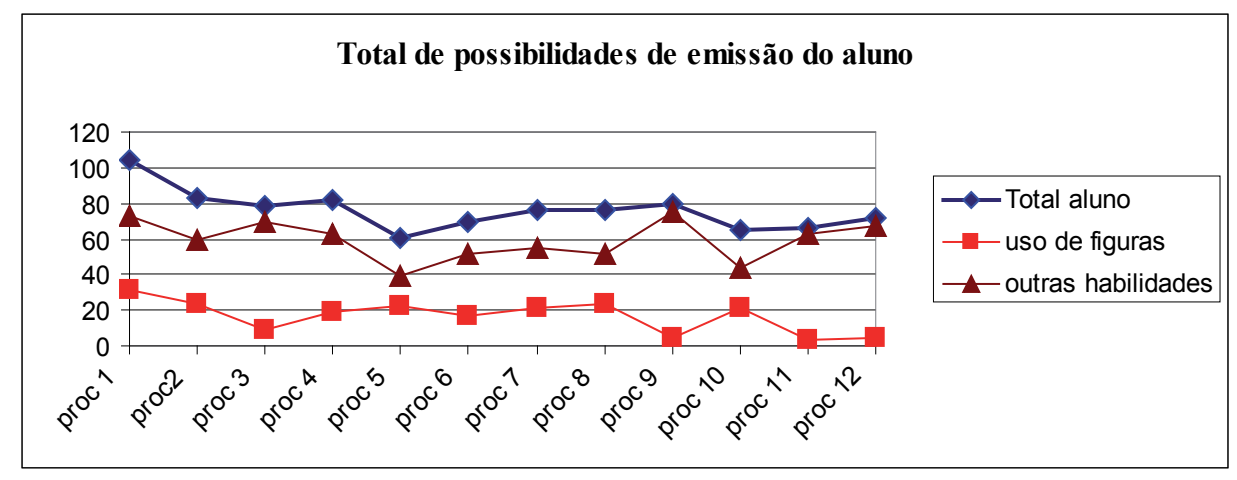

Gráfico 1 - Número de possibilidades expressivas utilizadas pelo aluno, durante os 12 procedimentos

No procedimento 9, foi possível perceber que o aluno utilizou com menor freqüência as figuras do seu tabuleiro de comunicação, provavelmente em função do tema desenvolvido: conserto do tabuleiro. Neste contexto, o aluno utilizou, com maior freqüência, outras habilidades expressivas, como o uso do olhar para o próprio objeto, movimentação com a cabeça e o uso das mãos para representar as ações ocorridas, como pode ser observado a seguir:

Exemplo 4: $9^{\circ}$ procedimento

Categoria-alvo: habilidades expressivas de A. 
Descrição do contexto: T1, T2 e A. estão conversando a respeito do tabuleiro.

(T1) Com quem ficou o tabuleiro?!

(A) A movimenta a cabeça de um lado para o outro e direciona o olhar para T1 (expressão não-oral - expressão facial - direcionamento de olhar para os lados/ espacial + expressão não-oral - expressão facial - direcionamento de olhar para T1)

(T1) Foi comigo??

(A) A. faz movimento de negação com a cabeça. (movimento de cabeça de negaç̧ão)

(T1) Não! Não foi comigo, não! (gesto de negação)

(A) A. permanece olhando para T1 + sorriso (expressão não-oral - expressão facial direcionamento de olhar para interlocutor + sorriso) ((concordar com a resposta))

(T1) Quem foi? Esqueceu?

(T2) Acho que ele esqueceu mesmo...

(A) A. direciona o olhar para baixo e a cabeça também + sorriso (direcionamento de olhar para baixo + movimento de cabeça para baixo $)$ - ((não quer responder $))$.

(T1) O que foi??

(A) A. direciona o olhar para T1 e faz com os dedos indicadores o desenho de um quadrado em cima da mesa adaptada (direcionamento de olhar para $\mathrm{T1}+$ desenho de quadrado na mesa) ((tabuleiro)).

(T1) É!! Isso mesmo, o tabuleiro (gesto do formato do tabuleiro), aquele que tem um monte de figuras e que nos ajuda a conversar. Com quem será que ficou?

(A) A. novamente direciona a cabeça e o olhar para baixo. (direcionamento de olhar para baixo) - ((não quer responder)).

(T1) Com quem será que está esse tabuleiro???

(A) A. direciona o olhar para T1 + sorriso, e indica com a cabeça para T1 (direcionamento de olhar para T1+ movimento de cabeça para localizar a pessoa).

Nos procedimentos 11 e 12, apesar de o aluno estar com o tabuleiro aberto, utilizou habilidades combinadas para relatar experiências pessoais orais, nãoorais ou somente as habilidades combinadas não-orais, como pode ser visualizado a seguir:

\section{Exemplo 8: $11^{\circ}$ procedimento}

\section{Categoria-alvo: habilidades expressivas combinadas} tabuleiro.

Descrição do contexto: T1, T2 e A. estão conversando a respeito do

(T1) Mostra aqui para mim, A. (enquanto falava, T1 indicava o tabuleiro que já estava em cima da mesa adaptada).

(A) A. permanece apontando a caixa que está na mão de T1, com o dedo indicador (expressão não-oral - movimento com as mãos - indicação de objeto/caixa).

(T1) Mudou a caixa, né...

(A) direciona o olhar para T1 e pisca os olhos (expressão não-oral - expressão facial direcionamento de olhar + piscar).

(T1) A., de quem você ganhou esta gaita? 
(A) A. direciona o olhar para o tabuleiro de comunicação + emissão ininteligível (expressão não-oral - expressão facial - direcionamento de objeto/tabuleiro + emissão ininteligivel)

(T1) Foi da sua avó?

(A) A. direciona o olhar para T1 e fala [não] + [ubão]. (expressão não-oral - expressão facial-direcionamento de olhar para T1+ [não]+[ubão])

(T1) Ah! Foi do pai que você ganhou. Você ganhou de presente de aniversário?

(A) A. permanece olhando para T1 + sorriso (expressão não-oral - expressão facial direcionamento de olhar para $\mathrm{T} 1+$ sorriso).

(T1) Fala para mim, de quem foi?

(A) A. olha para o tabuleiro + emissão de som (expressão não-oral - expressão facial - direcionamento de olhar objeto/tabuleiro + emissão ininteligível)

(T1) Então, vamos lá.

(A) A. direciona o olhar para o que está sendo pego por T1. (expressão não-oral expressão facial - direcionamento de olhar para objeto/gaita).

(T1) Então, vamos ver se você sabe tocar esta gaita. Primeiro, tem que engolir a saliva.

(A) A. direciona o olhar para $\mathrm{T} 1+$ sorriso \{enquanto isso, a cadeira de A. se movimenta\} (expressão não-oral - expressão facial - direcionamento de olhar para $\mathrm{T} 1+$ sorriso).

Nos procedimentos 11 e 12 foi observado que o aluno utilizou, com menor frequência, o tabuleiro de comunicação, mas foi possível ser entendido pelos demais interlocutores em decorrência das suas habilidades não-verbais. Os temas desenvolvidos nos procedimentos e o conhecimento prévio dos demais interlocutores participantes a respeito da atividade e do aluno favoreceram as trocas comunicativas sem o uso do tabuleiro de comunicação. A literatura da área tem discutido a interferência do interlocutor no uso das habilidades expressivas do usuário de comunicação suplementar e alternativa, principalmente se o interlocutor faz parte da rotina da criança e jovem com deficiência (NUNES, 2003a, b; FERM; AHLSÉN; BJORCK-AKESSON, 2005; DELIBERATO, 2005; SAMESHIMA; DELIBERATO, 2009; DELIBERATO; ALVES, 2009).

No procedimento 11, o aluno usou somente três indicações de figuras, não só pelo motivo do uso das demais habilidades, mas também pelo manuseio do objeto trazido de casa, a gaita.

Nas situações dialógicas estabelecidas entre os interlocutores, o aluno participou das trocas de turnos por meio de diferentes modalidades expressivas. $\mathrm{O}$ uso do sistema gráfico, quer na estrutura vertical, quer associado à outra modalidade de expressão, esteve nas situações dialógicas por parte do aluno selecionado para esta pesquisa. O Gráfico 2 demonstra a ocorrência do uso do sistema gráfico em relação às demais habilidades expressivas, nas situações dialógicas assumidas pelo aluno: 


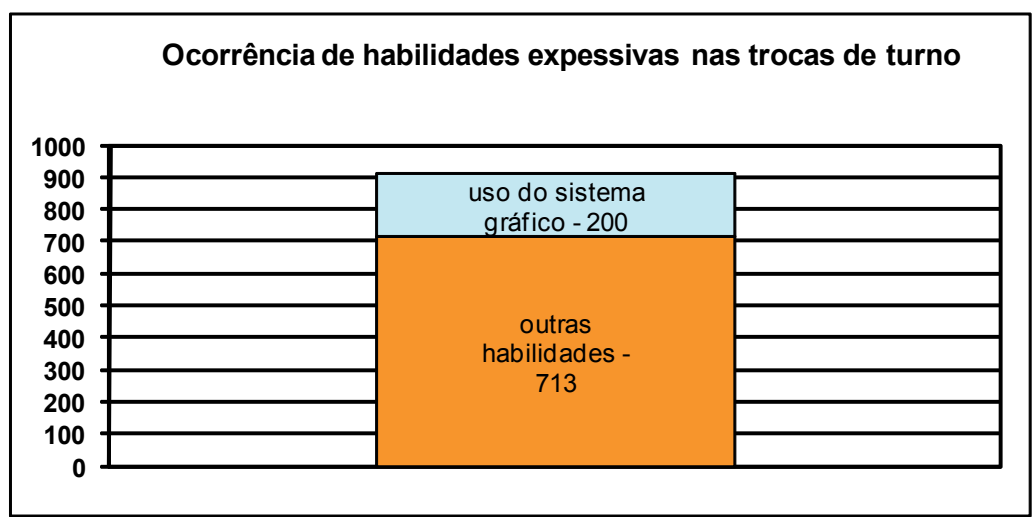

Gráfico 2 - Ocorrência das modalidades expressivas nas trocas de turnos

Convém ressaltar que o uso do sistema gráfico ocorreu na estrutura vertical como já definido anteriormente, ou seja, o uso de um único símbolo gráfico para a transmissão de uma mensagem, quer simples ou complexa e, na estrutura horizontal, que foi a ocorrência da combinação de mais de um símbolo gráfico; ou ainda, a combinação de símbolo gráfico com outras habilidades expressivas, constituindo, dessa forma, elementos de uma organização sintática. O Gráfico 3, a seguir, evidencia a ocorrência do símbolo gráfico na estrutura vertical e com outras habilidades expressivas, compondo estruturas diversificadas de enunciados.

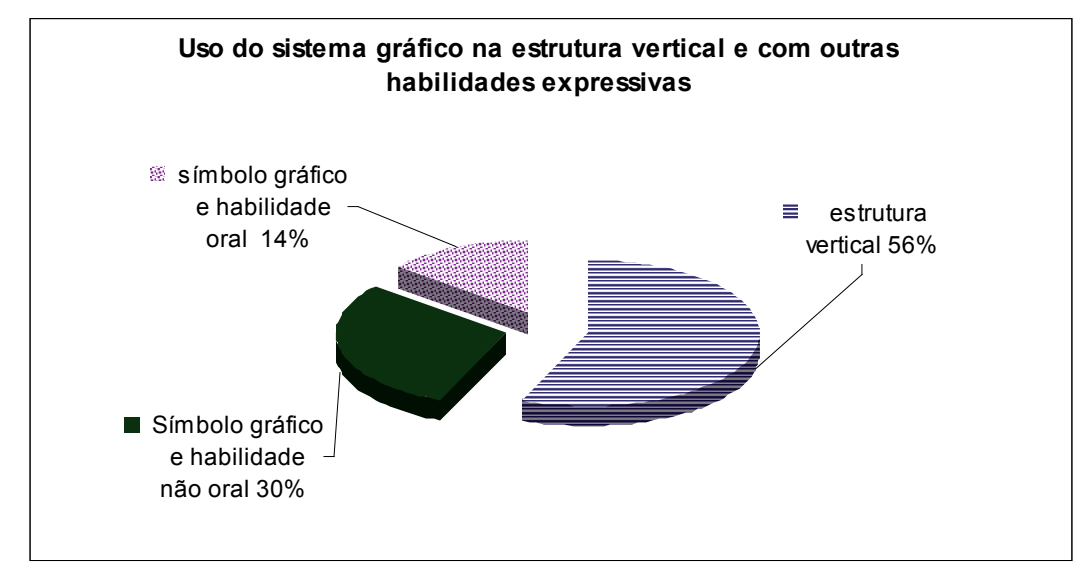

Gráfico 3 - Ocorrência do sistema gráfico nas habilidades expressivas

Como é possível observar, o aluno utilizou com maior frequência o símbolo gráfico na estrutura vertical, seguida pelo uso associado à habilidade expressiva não-oral. Para Udwin e Yule (1990), Von Tetzchner e Martinsen (1996) e Light (1997), crianças e jovens usuários de sistemas gráficos com boa compreensão 
verbal tendem a usar enunciados com um só símbolo, isto é, as pessoas com recursos de comunicação suplementares e alternativos de comunicação utilizam a estrutura vertical independente da fala e da idade (NUNES et al., 2003). Tal fato poderia estar sendo influenciado pelas experiências linguísticas e de aprendizagem serem diferentes dos demais indivíduos que possuem a possibilidade de falar (BEUKELMAN; MIRENDA, 2007).

Durante os 12 procedimentos, o aluno adotou o sistema gráfico por meio da seleção do olhar, do gesto indicativo e ainda pela movimentação de cabeça. A seleção do sistema foi feita como elemento único na situação dialógica ou ainda associada a outras habilidades expressivas, quer orais, quer não-orais, ou ainda pelo acesso de mais de um símbolo gráfico na sequência, como já foi pontuado anteriormente.

O Gráfico 4, a seguir, demonstra o acesso ao sistema gráfico, na situação dialógica realizada pelo aluno:

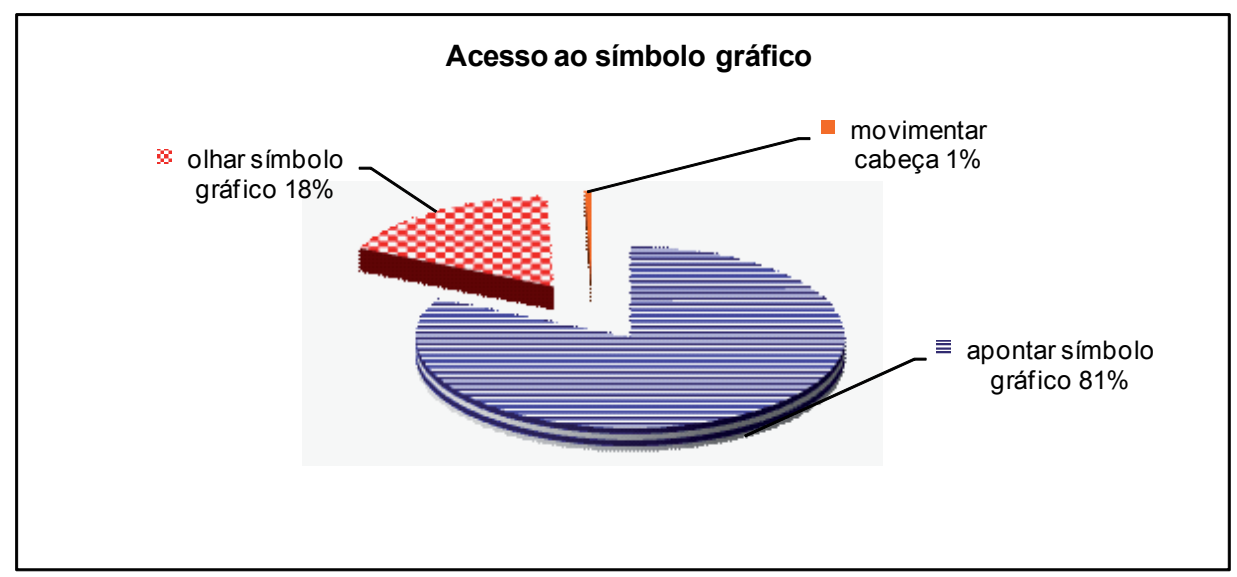

Gráfico 4 - Acesso ao sistema gráfico durante os 12 procedimentos

A circunstância de o aluno ter condições físicas para acessar o tabuleiro de comunicação favoreceu o uso dos símbolos por meio do gesto de apontar com o dedo seguido pelo olhar. Esse aspecto foi um dos domínios salientados por Light (2003) como responsáveis pelo sucesso no uso do sistema de comunicação suplementar e alternativa: o domínio operacional.

Como foi observado no Gráfico 3, o aluno empregou o sistema gráfico nas situações dialógicas com maior ocorrência na estrutura vertical, segundo se pode notar a seguir:

Exemplo 5: $5^{\circ}$ procedimento da figura

Categoria-alvo: subcategoria da habilidade de expressão não-oral - uso 
Descrição do contexto: relato de passeio

(T1) A., fala para mim onde o peixe estava?

(A) A. indica uma figura PAI. (expressão não-oral - movimento de mão - indicação de figura PAI)

(T1) Pai? O que você fez com o seu pai? (com apoio das figuras)

(A) A. indica outra figura no tabuleiro, CARRO. (expressão não-oral - movimento de mão - indicação de figura CARRO).

(T1) Carro, pai? Aonde você foi com o seu pai? (com apoio das figuras)

(A) A. faz gesto com as mãos, uma na frente da outra, indo para frente (expressão não-oral - gesto representativo com as mãos: expressando movimento para frente).

(T1) Vocês foram embora para onde?

(A) A. indica mais uma figura, AVÔ. (expressão não-oral - movimento de mão indicação de figura AVÔ).

(T1) Avô? Você e seu pai foram de carro (gesto) à casa do avô? (T1 usa o apoio das figuras).

(A) A. faz movimento de cabeça, afirmando (movimento de cabeça afirmativa).

(T1) Agora sim, eu entendi! Mas e o peixe, você ainda não me respondeu onde ele estava...

(A) A. indica a figura RIO. (indicação de figura RIO).

Por meio do exemplo 5, é possível identificar uma fala telegráfica, ou seja, o aluno manteve o diálogo utilizando um símbolo único e foi ampliando as respostas pela mediação da terapeuta. Durante essa mediação, ocorreu a sequência PAI + CARRO + movimento das mãos para frente ((ir)) + AVÔ. No modelo oferecido pela terapeuta, pela fala e as figuras, o enunciado aceito pelo aluno foi: você e seu pai foram de carro na casa do seu avô. Cabe ressaltar o uso do gesto representativo, para o aluno atribuir a ação realizada por ele e seu pai.

Kelford-Smith et al. (1989); Udwin e Yule, 1990; Soto e Toro-Zambrana (1995); Smith (1996) e Sutton et al. (2000) identificaram em crianças, jovens e adultos usuários de comunicação suplementar e alternativa os enunciados reduzidos, quando comparados com as sentenças faladas, mesmo que as pessoas submetidas aos estudos tivessem características diferentes e os sistemas fossem diferenciados. Embora os enunciados do aluno participante, tenha acontecido de forma reduzida e telegráfica, o sistema gráfico permitiu a essa criança uma atitude ativa, na situação dialógica (DE COSTE, 1997).

A expansão de enunciados por meio dos sistemas gráficos é discutida na literatura como um dos aspectos fundamentais para o trabalho direcionado à linguagem. Von Tetzchner (2009) advertiu que, devido ao fato de o vocabulário disponível nos sistemas gráficos ser, em geral, limitado, é importante que os adultos (cuja fala é modelo, na língua materna) expandissem os enunciados do usuário de CSA, de tal modo que este se tornasse apto a produzir, pelo menos, parte do enunciado expandido, naquele sistema. 
O exemplo a seguir ilustra a participação do aluno na situação dialógica, com apoio do sistema gráfico ampliando seu enunciado:

Exemplo 6: $5^{\circ}$ procedimento

Categoria-alvo: habilidade expressiva não-oral e verbal - sistema gráfico

Descrição do contexto: alimentação

(A) A. direciona o olhar para T1, faz movimento de cabeça, erguendo-a e indo para trás. Em seguida, A. aponta uma figura no tabuleiro TERAPEUTA. (expressão nãooral - expressão facial - direcionamento de olhar para trás/espacial + figura TERAPEUTA)

(T1) Eu? O que tenho eu, A.? Mostra aqui no tabuleiro.

(A) A. indica com o dedo, no tabuleiro, a figura PEIXE.

(T1) Peixe? Eu não estou entendendo, A.

(A) A. faz o gesto de comer com as mãos e indica TERAPEUTA + PEIXE (gesto representativo de comer + expressão não-oral - movimento de mão - indicação de figura TERAPEUTA + PEIXE)

(T1) Comer, eu, peixe? Não entendi!

(A) A. indica com o dedo a figura GOSTAR + direção de olhar para T1. (Figura GOSTAR + expressão não-oral - expressão facial - direcionamento de olhar para interlocutor)

(T1) Ah!! Agora eu entendi. Você está perguntando se eu gosto de comer peixe? (seguindo o modelo com os símbolos do tabuleiro)

(A) A. fala [é] + movimento de cabeça afirmando. ([é] + movimento de cabeça afirmando).

(T1) Eu gosto, e você gosta?

(A) A. faz que sim com a cabeça + indicação de outra figura no tabuleiro COMER e direciona o olhar para T2. (movimento de cabeça afirmando + figura COMER+ expressão não-oral - expressão facial - direcionamento de olhar T2).

(T1) T2, o que tem o T2?

(A) A. indica uma figura COMER + sorriso. (expressão não-oral - movimento de mão - indicação de figura COMER +sorriso)

(T1) Comer?? Ah! Se o T2 gosta de comer peixe? (com apoio das figuras do tabuleiro)

(A) A. faz movimento de cabeça afirmando + sorriso. (expressão não-oral - movimento de cabeça afirmando)

(T1) Ele adora. Ele gosta muito (gesto) de comer peixe, os dois: eu e ele. (Enquanto falava, indicava as figuras no tabuleiro)

O exemplo 6 leva a perceber que o aluno, no primeiro momento da pergunta para T1, utilizou um símbolo; após a mediação da terapeuta, usou o gesto representativo para a ação comer e, em seguida, a indicação de dois símbolos gráficos consecutivos: TERAPEUTA + PEIXE, ampliando o enunciado. Dessa forma, a frase seria: comer + TERAPEUTA + PEIXE. Depois disso, a terapeuta dá continuidade à mediação: “Comer, eu, peixe? Não entendi!” Após a intervenção de $\mathrm{T} 1$, o aluno selecionou outro símbolo, ampliando o enunciado e garantindo o real sentido: GOSTAR, de maneira que o enunciado ficaria: comer + TERAPEUTA + 
PEIXE + GOSTAR. Logo em seguida, T1 oferece o modelo adequado de organização frasal: Você está me perguntando se eu gosto de comer peixe?

Nunes et al. (2003), Von Tetzchner (2009) e Von Tetzchner e Grove (2003) afirmaram ser recomendável que modelos de organização sintática da linguagem oral sejam fornecidos ao usuário de CSA, como forma alternativa de expressão, aceitando-se, porém, a ordenação desviante. Tal fato pode ser observado no momento em que o aluno seleciona a figura COMER e direciona o olhar para T2. Nesse momento, T1 não percebeu a indicação de a figura COMER e questionou o que significava o direcionamento de olhar para T2. A. aponta novamente a figura COMER + sorriso, respondendo a respeito da intenção do olhar para T2.

Nessa situação, fica salientada a importância da habilidade expressiva não-oral, o direcionamento de olhar, que, embora não-verbal, contribuiu para compreender o sentido da intenção do aluno: Ele (T2) come? O alimento PEIXE ficou subentendido no contexto da situação dialógica.

A criança e o jovem usuário de sistemas de comunicação suplementar e alternativa podem empregar diferentes habilidades expressivas (verbais; nãoverbais; vocal/oral; não-vocal/oral) com ou sem auxílio, mas devem estar atentos à escolha da habilidade expressiva, para que o interlocutor possa compreendê-los. Assim, a literatura reforçou que os sistemas de representação, como os símbolos gráficos, poderiam garantir o entendimento por parte do interlocutor, conforme se vê no exemplo 7:

\section{Exemplo 7: $5^{\circ}$ procedimento} da figura.

Categoria-alvo: subcategoria da habilidade de expressão não-oral - uso

Descrição do contexto: relato de passeio

(T1) Você já andou a cavalo?

(A) A. faz movimento de cabeça afirmando + sorriso (expressão não-oral - movimento de cabeça afirmando + sorriso)

(T1) Onde você andou?

(A) A. ergue a cabeça e faz movimento giratório para trás + sorriso. (Movimento de cabeça para trás/indicação espacial (indicar escola/casa + sorriso).

(T1) Onde? Eu não sei o que é esse movimento de cabeça, fala aqui para mim, onde? (enquanto falava, indicava o tabuleiro)

(A) A. indica a figura da PROFESSORA

(T1) Professora? Ah! Você andou a cavalo com a professora?

(A) A. fala [não] e bate a mão direita em cima da mesa + franzimento de sobrancelha ([não] + bate a mão na mesa/bravo+movimento de sobrancelha)

(T1) Então, fala para mim.

(A) A. indica com o dedo a figura ESCOLA

(T1) Agora eu entendi, você foi num passeio com a escola. (com apoio das figuras do tabuleiro) 
(A) A. faz movimento de cabeça afirmando + direção de olhar para T1. (movimento de cabeça afirmando + expressão não-oral - expressão facial - direcionamento de olhar para interlocutor).

No exemplo 7, o aluno utilizou a figura da PROFESSORA para substituir a habilidade expressiva feita com o movimento de cabeça (movimento feito com a cabeça em direção para trás), para dar sentido à intenção de que ele foi passear a cavalo com a escola. A figura da professora foi o símbolo mais imediato a ser indicado, mas o interlocutor também não compreendeu o sentido atribuído pelo aluno. Frente à situação, o aluno indicou a figura mais próxima: ESCOLA, para dar continuidade à situação dialógica e, dessa forma, atingir o interlocutor.

Smith (1996) identificou o uso de um mesmo símbolo para representar mais de um significado a fim de que o usuário do sistema gráfico possa ser mais rápido e funcional; muito embora Muniz (2004) tenha relatado, em sua pesquisa, que, ao utilizar um mesmo símbolo para significados diferentes, o interlocutor presente não entendeu a estratégia adotada pela criança com paralisia cerebral, conforme ocorreu no exemplo citado.

Nunes et al. (2003) estudaram estratégias como a solicitação de clarificação, o mando para a extensão, a síntese e o uso de modelos, as quais poderiam facilitar aos usuários de comunicação suplementar e alternativa a compreensão de que um determinado símbolo gráfico nem sempre é suficiente para expressar a intenção pretendida e, com isso, ser apreendido pelos diferentes interlocutores.

McCleary e Smith (2006) abordaram a dificuldade em sistematizar as modalidades de expressão usadas por crianças com impedimento da linguagem falada. As autoras perceberam que a própria criança e seus familiares conduziram à escolha mais funcional. Nessa mesma direção, Gulens, Kerbel e Nobel (2006) enfatizaram a necessidade de os profissionais estarem atentos aos tipos de estratégias a serem realizadas com crianças e jovens com severo comprometimento de comunicação, inserindo, nos programas, as habilidades comunicativas funcionais já existentes.

\section{CONCLUSÃo}

O emprego de habilidades não-orais e verbais, como sistemas de comunicação suplementares e alternativos, favoreceu a participação do aluno na situação dialógica. Com relação ao uso das mãos, o gesto indicativo foi a habilidade mais frequente, nos 12 procedimentos, sobretudo para o uso do sistema gráfico, ou seja, o uso da expressão não-oral e verbal. O direcionamento de olhar também foi empregado como possibilidade de indicar objetos (28\%) e elementos do sistema gráfico $(13 \%)$, entre as modalidades de direcionamento de olhar. $\mathrm{O}$ aluno usou $\mathrm{o}$ sistema gráfico na estrutura vertical (56\%), associado à modalidade oral (13\%) e à modalidade não-oral (verbal e não-verbal) $(30 \%)$ e, finalmente o uso do sistema 
gráfico em conjunto com outras modalidades ajudou na ampliação dos enunciados e na possibilidade de o aluno ser compreendido na sua intenção.

\section{Considerações Finais}

Os resultados, aqui identificados, podem contribuir e ampliar as ações dos profissionais, principalmente do fonoaudiólogo, com a criança e jovem com paralisia cerebral e possibilitar a sua inserção na comunicação suplementar e alternativa e nas possibilidades comunicativas que integram a linguagem. Embora esta pesquisa tenha limitações, principalmente em relação ao número de participantes e sua idade, no início da proposta do programa, o estudo poderá cooperar quanto à necessidade do envolvimento do fonoaudiólogo no programa de intervenção para o cliente, interlocutores nas tarefas a serem desenvolvidas e nos diferentes ambientes (SCHLOSSER; ROTHSCHILD, 2001; DELIBERATO, 2010).

O aluno participante desta pesquisa mostrou, durante os 12 procedimentos realizados com o recurso de comunicação suplementar e alternativa, a diversidade de possibilidades de manifestações expressivas, em busca de ser compreendido e manter sua atuação na situação dialógica. Foi possível perceber o maior número de ocorrências de habilidades expressivas não-orais e não-verbais, embora o uso das habilidades expressivas por meio de um sistema de representação tenha colaborado com a ampliação do enunciado e sentido atribuído pelo participante, na sua intenção.

\section{REFERÊNCIAS}

BARDIN, L. Análise de conteúdo. Tradução de Luís Antero Reto e Augusto Pinheiro. 3. ed. Lisboa: Edições 70, 2004.

BEUKELMAN, D. R.; MIRENDA, P. Augmentative \& alternative communication: supporting children \& adults with complex communication needs. Baltimore: Paul H. Brookes Publishing, 2007.

BLAU, A. F. A response to Fuller and Lloyd: toward an augmentative and alternative communication symbols taxonomy: a proposed superordinate classification. Augmentative and Alternative Communication, Vancouver, v. 3, n.2, p. 97-99, 1987.

CAPOVILLA, F. C.; DUDUCHI, M.; MACEDO, E. C. Sistema de multimídia para a comunicação picto-silábica: análise de seis meses de uso domiciliar de ImagoVox por mulher com paralisia cerebral. Ciência Cognitiva: Teoria, Pesquisa e Aplicação, São Paulo, v. 2, n. 3, p. 21-112, 1998.

CARVALHO, A. M. P. O uso do vídeo na tomada de dados: pesquisando o desenvolvimento do ensino em sala de aula. Pro-posições, v. 7, n. 1, p. 5-13, 1996.

COOK, A.; HUSSEY, S. Assistive technologies: principles and practice. 2. ed. New York: Mosby, 2002. 
DE COSTE, D. C. AAC and individuals with physical disabilities. Augmentative an Alternative Communication Assessment Strategies. In: GLENNEN, S. L.; DE COSTE, D. C. (Ed.). The handbook of argumentative and alternative communication. San Diego: Singular, 1997. p. 363-389.

DELIBERATO, D. Caracterização das habilidades expressivas de um aluno usuário de comunicação alternativa durante intervenção fonoaudiológica. 2010. 178 f. Tese de LivreDocência - Faculdade de Filosofia e Ciências, Universidade Estadual Paulista, Marília, 2010.

Speech and language therapy in the school: resources and procedures for augmentative and alternative communication. In: VON TETZCHNER, S.; GONÇALVES, M. J. (Ed.). Theoretical and methodological issues in research on augmentative and alternative communication. Vancouver: ISAAC, 2005. p. 116-125.

. Uso de expressões orais durante a implementação do recurso de comunicação suplementar e alternativa. Revista Brasileira de Educação Especial, Marília, v.15, p.369-388, 2009.

. Acessibilidade comunicativa no contexto acadêmico. In: MANZINI, E. J. (Org.). Inclusão do aluno com deficiência na escola: os desafios continuam. Marília: ABPEE, 2007. p.25-36.

DELIBERATO, D.; ALVES, V. A. Interação do aluno com deficiência sem oralidade frente a diferentes interlocutores. Educação em Questão, Natal, v. 34, p.102-126, 2009.

DELIBERATO, D.; MANZINI, E. J. Identification of the communicative abilities of children and young adults with cerebral palsy for the implementation of augmentative and alternative communication resources in the familiar context. In: BIENNIAL CONFERENCE OF THE INTERNATIONAL SOCIETY FOR AUGMENTATIVE AND ALTERNATIVE COMMUNICATION, 13., Montreal. Conference Proceedings..., Montreal: ISAAC, 2008. p. 104-105.

. Comunicação alternativa e aumentativa: delineamento inicial para a implementação do Picture Communication System (P.C.S.). Boletim do Centro de Orientação Educacional (COE), Marília, v. 2, p. 29-39, 1997.

FERM, U.; AHLSÉN, E.; BJORCK-AKESSON, E. Conversational topics between a child with complex communication needs and her caregiver at mealtime. Augmentative and Alternative Communication, Vancouver, v. 20, n. 1, p. 19-40, March 2005.

FAGUNDES, A. J. F. M. Descrição, definição e registro de comportamento. São Paulo: Edicon, 1983.

GULENS, M.; KERBEL, S.; NOBEL, L. Just talk: practical strategies for developing functional communication using AAC. In: BIENNIAL CONFERENCE OF THE INTERNATIONAL SOCIETY FOR AUGMENTATIVE AND ALTERNATIVE COMMUNICATION, 12., 2006. Conference Proceedings Duesseldorf: ISAAC, 2006. p. 209-211.

HIGGINBOTHAM, D. J. et al. Acess to AAC: present, past, and future. Augmentative and Alternative Communication, Vancouver, v. 23, n.3, p. 243-257, 2007.

JOHNSON, R. Boardmaker: The P.C.S. libraries on disk. Solana Beach: Mayer Johnson, 1994. 
KELFORD-SMITH, M. et al. The form and use of written communication produced by physically disabled individuals using microcomputers. Augmentative and Alternative Communication, Vancouver, v. 5, p. 115-124, 1989.

LIGHT, J. C. Development of communicative competence by individuals who use AAC. In: LIGHT, J. C.; BEUKELMAN, D. R; REICHLE, J. (Org.). Communicative competence for individuals who use AAC: from research to effective practice. Baltimore: Paul H. Brookes Publishing, 2003. p. 3-38.

"Let's go start fishing": Reflections on the contexts of language learning for children who use aided AAC. Augmentative and Alternative Communication, Vancouver, v.13, n. 2, p. 158-171, 1997.

LIGHT, J. C.; BINGER, C.; SMITH, A.K. Story reading interations between preschoolers who use AAC and their mothers. Augmentative and alternative communication, v.10, n.4, p.255-268, 1994.

MCCLEARY, M.; SMITH, M. Communication modes, then and now: case studies. In: BIENNIAL CONFERENCE OF THE INTERNATIONAL SOCIETY FOR AUGMENTATIVE AND ALTERNATIVE COMMUNICATION, 12., 2006. Conference Proceedings ..., Duesseldorf: ISAAC, 2006. p. 423.

MILLIKIN, C. Symbol system and vocabulary selection strategies. In: GLENNEN, S.; DeCOSTE, D. C. (Ed.). The handbook of augmentative and alternative comunication. San Diego: Singular Publishing Group, 1997. p. 97-148.

MUNIZ, F. B. Narrativa de um aluno com paralisia cerebral por meio do uso de recursos e estratégias de comunicação suplementar. 2004. 121 f. Dissertação (Mestrado em Educação) Faculdade de Filosofia e Ciências, Universidade Estadual Paulista, Marília, 2004.

NUNES, L. R. O. P. Linguagem e comunicação alternativa: uma introdução. In: NUNES, L. R. O. (Org.). Favorecendo o desenvolvimento da comunicação em crianças e jovens com necessidades educacionais especiais. Rio de Janeiro: Dunya, 2003a. p. 1-13.

. Modelos teóricos na comunicação alternativa e ampliada. In: NUNES, L. R. O. (Org.). Favorecendo o desenvolvimento da comunicação em crianças e jovens com necessidades educacionais especiais. Rio de Janeiro: Dunya, 2003b. p. 15-47.

NUNES, L. R. O. P. et al. Narrativas sobre fotos e vídeos e narrativas livres através de sistema gráfico de Comunicação Alternativa. In: NUNES, L.R.O.P. (Org.). Favorecendo o desenvolvimento da comunicação em crianças e jovens com necessidades educacionais especiais. Rio de Janeiro: Dunya, 2003. p. 143-169.

SAMESHIMA, F. S.; DELIBERATO, D. Habilidades expressivas de um grupo de alunos com paralisia cerebral na atividade de jogo. Revista da Sociedade Brasileira de Fonoaudiologia, São Paulo, v. 14, p. 219-224, 2009.

SCHLOSSER, R.; ROTHSCHILD, N. Augmentative and alternative communication for persons with developmental disorders. Temas sobre Desenvolvimento, São Paulo, v. 10, n. 58-9, p.6CE-17CE, 2001. 
DELIBERATO, D.

SMITH, M. M. The medium or the message: A study of speaking children using communication boards. In: VON TETZCHENER, S.; JENSEN, M. H. (Ed.). Augmentative and alternative communication: european perspectives. London: Whurr, 1996. p. 119-136.

SMITH, M. M.; GROVE, N. Asymmetry in input and output for individuals who use AAC. In: LIGHT, J. C.; BEUKELMAN, D.R.; REICHLE, J. (Org.). Communicative competence for individuals who use AAC: from research to effective practice. Baltimore: Paul H. Brookes Publishing, 2003. p. 163-195.

. The bimodal situation of children learning language using manual and graphic signs. In: BIENNIAL CONFERENCE OF THE INTERNATIONAL SOCIETY FOR AUGMENTATIVE AND ALTERNATIVE COMMUNICATION. 7., 1996. Conference Proceedings ..., Vancouver: ISAAC, 1996. p. 134-137.

SORO-CAMATS, E. Uso de ajudas técnicas para a comunicação, o jogo, a mobilidade e o controle do meio: uma abordagem habilitadora. In: ALMIRALL, C. B.; SORO-CAMATS, E.; BULTÓ, C. R. (Org.). Sistemas de sinais e ajudas técnicas para a comunicação alternativa e a escrita: princípios teóricos e aplicações. São Paulo: Livraria Santos Editora, 2003. p. 23-41.

SOTO, G.; TORO-ZAMBRANA, W. Investigation of Blissymbol use from a language research paradigm. Augmentative and Alternative Communication, Vancouver, v. 11, n.2, p. 118-130, 1995.

SUTTON, A. et al. Relative clause sentence production using augmentative and alternative communication systems. Applied Psycholinguistics, Cambridge, v. 21, p. 473486, 2000.

UDWIN, O.; YULE, W. Augmentative communication systems taught to cerebral palsied children: a longitudinal study. The acquisition of the signs and symbols, and syntatic aspects of their use over time. British Journal of Communication Disorders, Edinburgh, v. 25, p. 295-309, 1990.

VON TETZCHNER, S. Suporte ao desenvolvimento da comunicação suplementar e alternativa. In: DELIBERATO, D.; GONÇALVES, M. J.; MACEDO, E. C. (Org.). Comunicação alternativa: teoria, prática, tecnologias e pesquisa. São Paulo: Memnon Edições Científicas, 2009. p. 14-27.

VON TETZCHNER, S.; GROVE, N. The development of alternative languages forms. In: (Ed.). Augmentative and alternative communication: developmental issues. London: Whurr, 2003. p. 1-27.

VON TETZCHNER; MARTINSEN. Words and strategies: communication with young children who use aided language. In: VON TETZCHNER, S.; JENSEN, M. H. (Ed.). Augmentative and alternative communication: European perspectives. London: Whurr, 1996. p. $65-88$.

\footnotetext{
Recebido em: 29/10/2010

Reformulado em: 13/06/2011

Aprovado em: 05/07/ 2011
} 